\title{
Experimental Tobacco Carcinogenesis
}

National Cancer Institute

\section{Source}

National Cancer Institute. Experimental Tobacco Carcinogenesis. NCI Thesaurus. Code C20122.

Studies on the toxicology and pharmacology of smoking and tobacco-related exposures.

The research in this category is directed toward understanding the nature of the chemicals involved, and the reaction mechanisms behind, the deleterious effects of tobacco smoke and smokeless tobacco products. 